\title{
The relative contribution of medical and surgical referrals to the workload in general ophthalmic practice
}

\section{K Greiner, K McCormack, A Grant, J V Forrester}

There needs to be a better estimate of the work currently done by surgical ophthalmologists

S urgical advances have been spectacular during the past few decades and have radically changed the practice of ophthalmology. ${ }^{1-3}$ Accordingly, most of the subspecialisation in ophthalmology is surgically oriented and the trainee in the 21 st century rightly devotes much time honing skills in surgical manoeuvres. However, all ophthalmologists are aware that a large part of practice relates to medical-that is, non-surgical, problems. The severity and management of these "medical" conditions vary. While the treatment of some is relatively straightforward, the treatment of others is more complex such that it becomes a major responsibility for those providing care to ensure that care is properly delivered and monitored. Many of these conditions are related to broader general medical problems, such as rheumatology, neurology, endocrinology and diabetology, cardiovascular disease, and inflammatory/infectious disease. In these circumstances, ophthalmic surgeons commonly request assistance in the medical care of these patients from a general physician. However, this places a significant burden on the physician who may not feel sufficiently qualified to monitor the responses to medical treatment of the eye.

This is the argument for a new kind of ophthalmologist who is skilled in ophthalmic medical diagnosis, who can relate systemic medical disease to ophthalmological conditions, who can assess the general medical status of patients who have a primary ophthalmological disease, and who can prescribe and monitor the most appropriate drugs for treatment of medical sight threatening disease. ${ }^{4}$ It is on this basis that a limited training programme for consultant specialist medical ophthalmologists is already in place in the United Kingdom. However, recruitment in this programme has been slow, the result in part of the limited number of consultant positions currently funded. Nevertheless, before medical ophthalmology posts can be made available by hospital trusts, there needs to be a better estimate of the work currently done by surgical ophthalmologists and of how much of this work could be taken over by a consultant medical ophthalmology (CMO) appointee. In this commentary we report an analysis of the types of newly referred adult patients attending a regional general ophthalmic unit. Our purpose was to investigate the specific medical and surgical diagnostic patterns among new referrals; to determine how often patients diagnosed with a medical or surgical condition required subsequent consultations and thus to estimate the workload which could be categorised as medical or surgical ophthalmology.

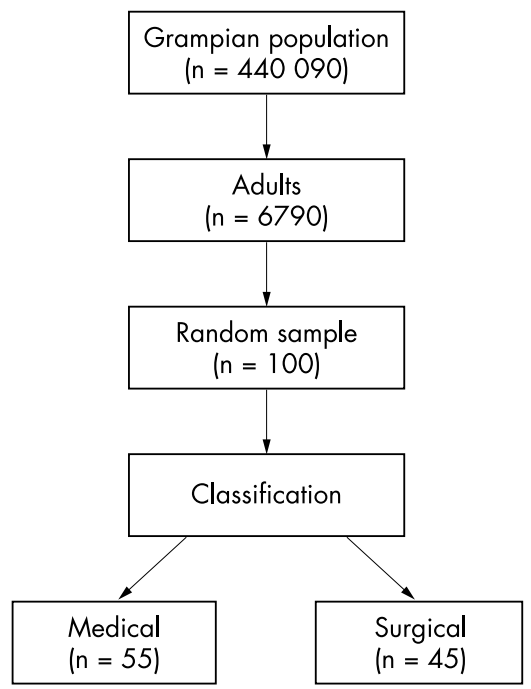

Figure 1 Sample characteristics.

We used routinely collected hospital patient administration system (PAS) data to identify all adults newly referred to the ophthalmology department of Grampian University Hospitals in Aberdeen over a 1 year period ( 1 June 1999 to 31 May 2000). These included general eye referrals and referrals to a specialist clinic. All referrals over the time period were given a randomly generated number, were sorted by this, and the first 100 chosen as a random sample. The hospital case notes of these patients were reviewed and data describing date of birth, sex, date of first service contact, diagnosis, and the number of subsequent outpatient appointments over a follow up period of 1 year were abstracted. The patients were categorised by the single diagnosis requiring the most immediate attention.

In the context of this study a surgical diagnosis was one judged to require a surgical opinion (even if surgery might not follow). It comprised the following groups:

Cataract

Glaucoma

Cornea/anterior segment

Vitreoretinal disease

Lacrimal/oculoplastics/orbit/oncology

Ocular motility.

A medical diagnosis was defined as any ocular problem for which surgery (other than laser therapy) would not be considered. The specific categories were: Diabetic eye disease

Medical retinal disease (including age related macular degeneration (AMD) and vascular disease)

Ocular inflammatory disease (including thyroid eye disease and connective tissue disease)

Neuro-ophthalmology.

Other.

We conjectured that about $50 \%$ of cases (rather than the large majority) would be medical. We decided to estimate this proportion with confidence intervals of plus or minus $10 \%$ and hence chose a sample size of 100 to give a standard deviation of $5 \%$. Data were entered into a database using Microsoft Access and analysed using SPSS (Statistical Package for the Social Sciences, Chicago, IL, USA). A study number was used to identify subjects, and person identification information was kept separately for the purpose of confidentiality. Simple descriptive statistics with 95\% confidence intervals for the estimated proportions were derived, where appropriate.

Over a period of 1 year a total of 6790 patients were referred directly to the eye clinic (general and specialist) for their first referral. Of these, 4268 (63\%) were referred by general practitioners, 1657 ( $24 \%$ ) by other consultants in Grampian University Hospitals Trust, and the remainder (13\%) were referred by consultants in other Grampian Health Board trusts or other health boards, accident and emergency department staff, or were referrals from penal establishments.

Among the random sample, 55 patients (55\%; 95\% CI 45 to 65 ) were diagnosed with a medical condition, and 45 (45\%; $95 \%$ CI 35 to 55$)$ with a surgical 
Table 1 Diagnostic categories of the random sample $(n=100)$

\begin{tabular}{lc}
\hline Categories & Number of patients (\%) \\
\hline Medical: & $5(9)$ \\
Diabetic eye disease & $20(36)$ \\
Medical retina (age related macular degeneration and vascular) & $16(29)$ \\
Ocular inflammatory disease (thyroid and connective tissue) & $8(15)$ \\
Neuro-ophthalmology & $5(9)$ \\
Ocular surface (non-inflammatory) & $1(2)$ \\
Other & No (\%) \\
Surgical: & $18(40)$ \\
Cataract & $6(13)$ \\
Glaucoma & $6(13)$ \\
Cornea/anterior segment & $9(20)$ \\
Vitreoretinal disease & $6(13)$ \\
Lacrimal/oculoplastics/orbit/oncology & $0(0)$ \\
Ocular motility &
\end{tabular}

condition (Fig 1). The diagnostic patterns and details of return visits within each group are given in Tables 1 and 2 . Within the medical diagnosis group the largest subgroup comprised patients with a medical retina problem, such as age related macular degeneration (AMD) and retinal vascular disease (36\%), followed by patients with ocular inflammatory disease (29\%) and diabetic eye disease (9\%). For patients with a surgical diagnosis the largest subgroup was that of cataract patients $(40 \%)$. The number of return visits ranged from 0 to 13 for those with a medical condition, and 0 to 12 within the surgical patient group (Table 2). Twenty three of 55 patients with a medical diagnosis $(42 \%)$ and 18 of 45 patients with a surgical diagnosis $(40 \%)$ did not require follow up appointments within 1 year of their first visit to the eye outpatient department. ity provides care representative of a UK regional service and serves a population of about 440000 and we believe that the hospital administration system used to identify patients provided a sound sampling frame for studying patterns of referrals. Our analysis suggests that 55\% of new patients referrals present with a medical problem as defined above (see Fig 1). Based on the 95\% confidence
The Grampian ophthalmological facil-

intervals, the true proportion is likely to be between $45 \%$ and $65 \%$. To put this another way, despite ophthalmology being a surgical specialty, it is unlikely that more than $55 \%$ of new referrals need to see an ophthalmic surgeon. Because of the size of our sample, some categories may be over-represented or under-represented by chance. Also, the types of referral to Grampian University Hospitals may differ from other hospitals. For example, the group with inflammatory eye disease may be somewhat over-represented here because Grampian University Hospitals acts as a tertiary referral centre with special interest in ocular inflammatory disease. Also, the proportion of newly referred patients with diabetic eye disease $(9 \%)$ in this study is likely to be lower than in district general hospitals. This is because diabetic patients are regularly seen and evaluated for diabetic eye disease in the physician led diabetic clinics, and are only referred to the eye department if sufficient (treatment requiring) severity of disease has been found. This, however, means that those referred represent a significant workload for the ophthalmologist (for example, they often require laser treatment or even vitrectomy).

At the time of the establishment of the NHS, almost all ophthalmologists were "general" ophthalmologists competent

\begin{tabular}{|c|c|c|}
\hline \multirow[b]{2}{*}{ Number of return visits } & \multicolumn{2}{|c|}{ Number of patients (\%) } \\
\hline & Medical diagnosis & Surgical diagnosis \\
\hline 0 & $23(42)$ & $18(40)$ \\
\hline 1 & $10(18)$ & $17(38)$ \\
\hline 2 & $6(11)$ & $5(11)$ \\
\hline 3 & $3(5)$ & $3(7)$ \\
\hline 4 & 6 (11) & 0 (0) \\
\hline 5 & $2(4)$ & $1(2)$ \\
\hline 6 & $1(2)$ & $0(0)$ \\
\hline 7 & $2(4)$ & $0(0)$ \\
\hline 12 & $1(2)$ & $1(2)$ \\
\hline 13 & $1(2)$ & $0(0)$ \\
\hline
\end{tabular}

to provide all aspects of ophthalmic care. However, during the past 25 years and with accelerating effect, there has been a progressive development of new diagnostic methods and refinement of treatment of ocular disease (for example, new immunosuppressive drugs for ocular inflammatory disease) paralleled by an increasing subspecialisation of ophthalmologists. ${ }^{1-35}$ This has been particularly so with respect to surgery with the advances in cataract surgery, vitreoretinal surgery, paediatric ophthalmology and ocular motility, and ophthalmic oncology as some representative examples. This has introduced a significant change in the balance of the delivery of ophthalmic care, particularly the greatly increased demand for cataract surgery, ${ }^{67}$ although there is some disagreement about this interpretation. ${ }^{8}$ The "general" ophthalmologist has become less common and many of the newly appointed consultant subspecialist ophthalmologists are engaged inappropriately in work that either does not require their expertise-for example, care of relatively straightforward medical problems in ophthalmology, or is outside their scope of interest and may thus prevent them from delivering the optimal treatment. Although some medical subspecialisation (for example, medical retina) has occurred and takes responsibility for a proportion of the medical ophthalmology patients, there is insufficient cover for a number of other medical diagnoses, such as sight threatening ocular inflammatory disease, diabetic eye disease, or neuro-ophthalmology.

Medical retina specialists do not typically possess expertise in treating some of the ocular inflammatory diseases (particularly posterior segment inflammation). Their usual domain is age related macular degeneration, retinal vascular disease, and inherited retinal disease. On the other hand, there are only few specialists dealing with ocular inflammatory disease, retinal vascular disease, and neuro-ophthalmology. Further, the medical ophthalmic workload is currently part of the responsibility of the surgical ophthalmologist whose time available for conducting surgical lists is thereby limited. This problem reflects itself most dramatically in the long waiting lists for cataract patients, ${ }^{6}{ }^{7}$ although there is disagreement about this interpretation. ${ }^{8}$ We suggest therefore that now may be an appropriate time to examine how we deliver ophthalmic services so that non-surgical referrals are referred to medical ophthalmologists and surgical ophthalmologists have greater opportunity to undertake surgical work. The introduction of the consultant medical ophthalmologist (consultant ophthalmic physician) has been a step in the right direction, but there 
would appear to be a need for a greater number of these individuals.

\section{ACKNOWLEDGEMENTS}

The Health Services Research Unit is supported by a core grant from the Chief Scientist Office of the Scottish Executive Health Department; however, the views expressed are those of the authors.

Br J Ophthalmol 2003;87:933-935

\section{Authors' affiliations}

K Greiner, J V Forrester, Department of Ophthalmology, Medical School, Aberdeen Royal Infirmary, Foresterhill, Aberdeen, UK
K McCormack, A Grant, Health Services Research Unit, University of Aberdeen, Polwarth Building, Foresterhill, Aberdeen, UK

Correspondence to: J V Forrester, Department of Ophthalmology, Medical School, Aberdeen Royal Infirmary, Foresterhill, Aberdeen AB25 2ZA, UK; j.forrester@abdn.ac.uk

\section{REFERENCES}

1 Williams PB, Crouch ER Jr, Sheppard JD Jr, et al. The birth of ocular pharmacology in the 20th century. J Clin Pharmacol 2000;40:990-1006.

2 Goh KY. Advances in ophthalmology. Singapore Med J 1999;40:563-4.

3 Adhikari T, Sarkar K, Datta H, et al. Revolutions in ophthalmology. J Indian Med Assoc 2000;98:770-1.
4 Dick AD, Forrester JV. The ophthalmic physician: an indulgence or a necessity? $\mathrm{Br} J$ Ophthalmol 1996;80:782-3.

5 Bourlais CL, Acar L, Zia H, et al. Ophthalmic drug delivery systems - recent advances. Prog Retin Eye Res 1998;17:33-58.

6 Churchill AJ, Vize CJ, Stewart OG, et al. What factors influence cataract waiting list time? Br J Ophthalmol 2000;84:429-3 1.

7 Minassian DC, Reidy A, Desai P, et al. The deficit in cataract surgery in England and Wales and the escalating problem of visual impairment: epidemiological modelling of the population dynamics of cataract. $\mathrm{Br} J$ Ophthalmol 2000;84:4-8.

8 Frost A, Hopper C, Frankel S, et al. The population requirement for cataract extraction a cross-sectional study. Eye $2001 ; 15: 745-52$.

FasL antibodies may compromise ocular immunity rather than cause eye disease

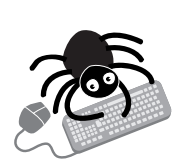

Please visit the British Journal of Ophthalmology website [www. bjophthalmol. com] for link to this full article.
- asL antibodies may compromise ocular immunity and so allow antibodies to corneal

- antigens to form, rather than directly causing eye disease, suggests a study of 34 patients with vasculitis.

The study was prompted by the knowledge that various corneal autoantigens have been found in patients with Wegener's granulomatosis and Churg-Strauss syndrome-most, but not all of whom, have peripheral ulcerative keratitis.

The presence of autoantibodies to FasL, K3, and BCEA-B was sought in 18 patients with Wegener's granulomatosis (WG) but no eye complications; seven with peripheral ulcerative keratitis (PUK); nine with Churg-Strauss syndrome (CSS); and in 14 people with no eye disease.

FasL antibodies were detected in two WG (11\%) and two PUK patients. Significantly, BCEA-B antibodies were also found in all four of these patients, but in only four (12.5\%) of the patients without FasL antibodies.

None of the patients positive for $\mathrm{K} 3$ antibodies carried antibodies to FasL, but one in four of those without FasL antibodies carried K3 antibodies. None of the patients with FasL antibodies had eye complications.

The authors conclude that it's not clear whether the apparent link between FasL antibodies and antibodies to ocular antigens is causal or coincidental. But they suggest that antibodies to FasL may block FasL function in the eye and compromise its ability to withstand inflammation and autoimmune disease.

A Ann Rheum Dis 2002;61:538-539. 